\title{
Labyrinthe
}

21 | 2005 (2)

Communauté en pièces : d'Europe, d'Islam et d'ailleurs

\section{Irène Rosier-Catach, La Parole efficace. Signe, rituel, sacré}

Avant-propos d'Alain de Libera, Seuil, coll. «Des Travaux», 2004, 704 p., $40 €$.

\section{Bérengère Hurand}

\section{(2) OpenEdition}

\section{Journals}

Édition électronique

URL : http://journals.openedition.org/labyrinthe/920

DOI : $10.4000 /$ labyrinthe.920

ISSN : 1950-6031

Éditeur

Hermann

\section{Édition imprimée}

Date de publication : 22 juin 2005

Pagination : 159-162

\section{Référence électronique}

Bérengère Hurand, « Irène Rosier-Catach, La Parole efficace. Signe, rituel, sacré », Labyrinthe [En ligne], 21 | 2005 (2), mis en ligne le 17 juillet 2008, consulté le 21 septembre 2020. URL : http:// journals.openedition.org/labyrinthe/920 ; DOI : https://doi.org/10.4000/labyrinthe.920 


\section{La Parole efficace. Signe, rituel, sacré d'Irène Rosier-Catach*}

Ego te baptizo, je te baptise: par cette simple formule, associée au geste d'immersion, le prêtre fait entrer l'enfant dans la communauté des chrétiens, sous la protection et l'amour de Dieu. Mais qui est cet ego ? De quel droit, et par quelle vertu opérative sa parole se substituet-elle à l'action divine ? Et la formule prononcée peut-elle être traduite, dite plusieurs fois, par deux personnes ou par le baptisé lui-même, sans que soit compromise la force de la grâce? Au Moyen Âge, les questions sur les sacrements ne manquent pas, et semblent même inventées à plaisir par leurs théoriciens; elles ont suscité les réflexions les plus diverses, dont témoignent les nombreux textes fondateurs, novateurs ou polémiques, rassemblés et traduits par Irène Rosier-Catach, directrice de recherche au CNRS, dans son ouvrage sur La Parole efficace. Du $\mathrm{XI}^{\mathrm{e}}$ au XIV $\mathrm{X}^{\mathrm{e}}$ siècle, de Bérenger de Tours à Duns Scot, la question a en effet mobilisé toutes les ressources logiques, grammaticales et sémiologiques disponibles, avec toute la constance et l'inventive obstination dont le Moyen Âge a été capable. La Parole efficace témoigne de cette minutieuse exploration; les multiples textes collectés, exclusivement théologiques, y sont analysés dans la complexité de leur agencement théorique, et travaillés, en rapport avec leur contexte historique, dans une double perspective épistémologique et philosophique. L'ouvrage dessine dans ses moindres détails une histoire médiévale du performatif, comme épisode désormais essentiel de l'histoire de la linguistique et de la sémiologie.

La richesse des théories médiévales sur le langage n'est plus à prouver. Il est essentiel, pour les théologiens médiévaux, de réfléchir sur la légitimité du discours sur Dieu, en même temps que de relayer adéquatement la parole divine elle-même et d'assurer l'opérativité, c'est-à-dire

\footnotetext{
* Avant-propos d'Alain de Libera, Seuil, coll. «Des Travaux», 2004, 704 p., $40 €$.
} 
l'efficacité des rituels. D'où la position centrale du sacrement. Comme serment, comme vœu, consentement au mariage ou formule eucharistique, c'est un énoncé performatif: il cause ce qu'il signifie (efficit quod figurat/significat). Décrit comme un «signe sacré» ou «signe de choses sacrées », le sacrement est aussi un processus sémiotique complet. Les formules sacramentelles, instituées par Dieu et par les croyants, sont codées; elles ont un énonciateur, un récepteur et des règles de fonctionnement. Le sacrement est donc pris dans une double dimension de signification et d'efficacité : en le traitant comme un simple signe, on juge sa valeur cognitive de vérité et sa capacité à renvoyer à autre chose que lui-même; en le traitant comme un acte, on évalue sa puissance opérative à effectuer ce qu'il désigne. Il s'agit alors, pour les théoriciens, d'examiner chaque élément du processus afin d'évaluer l'importance de son rôle dans l'acte, et sa responsabilité vis-à-vis de sa vertu opérative; de déterminer quel type de causalité régit l'efficacité du sacrement; et, plus généralement, d'observer le rapport que la formule sacramentelle établit d'une part avec son signifié, d'autre part avec son locuteur - qui est aussi son interprète.

Les voix des théologiens s'élèvent les unes après les autres dans $L a$ Parole efficace; parmi les plus illustres, on reconnaît celles d'Abélard et de Pierre Lombard au XII siècle, de Thomas d'Aquin, Bonaventure et Albert le Grand au XIII ${ }^{\mathrm{e}}$, de Duns Scot et Pierre de Poitiers au début du XIV ${ }^{e}$. Irène Rosier-Catach distingue deux courants principaux: les tenants de la «causalité-pacte» et ceux de la «causalité physique». Les uns, qui s'inspirent d'Augustin, insistent sur le rôle de l'intention dans l'efficacité sacramentelle, sur la valeur conventionnelle du rite et du pacte: tout comme les signes monétaires, les formules rituelles sont efficaces non quia dicitur, sed quia creditur - non parce qu'elles sont dites, mais parce qu'on y croit, et surtout parce qu'elles sont imposées. Les autres, influencés par Aristote, s'attachent plutôt aux propriétés intrinsèques des signes : la puissance du sacrement doit venir des mots eux-mêmes, et de leur association dans l'acte de prononciation. La convention seule ne peut leur conférer force d'obligation, puisqu'il n'est pas permis d'en modifier d'un iota la formule. On procède alors à des analyses logico-linguistiques pour légitimer l'opérativité des signes : la première personne, le présent, les déictiques ou la construction grammaticale des formules sont autant d'éléments irréductibles de la puissance quasi magique des paroles rituelles. Irène Rosier-Catach 
ne limite pas le débat aux temps obscurs du Moyen Âge théologique; elle rapporte cette hésitation de trois siècles sur le mode d'efficacité des sacrements à celle que résout Pierre Bourdieu au $\mathrm{XX}^{\mathrm{e}}$ quand il oppose, dans Ce que parler veut dire, sa conception d'une puissance exclusivement sociale de la parole à l'illusion des logiciens - pour lesquels la puissance de la parole est dans les mots eux-mêmes ${ }^{1}$.

Car la question du sacrement est aussi une question politique. Dans un contexte théologico-juridique où le discours fait autorité et où l'Autorité est le logos originaire, il importe en effet de savoir à quelles conditions un serment - comme le mariage ou le vœu religieux - est valable; c'est-à-dire à quelles strictes conditions la société peut considérer qu'une parole s'est matérialisée, qu'un acte irréversible a été posé dans la réalité politique. Pour dessiner le plus précisément possible les limites de validité du sacrement, les théologiens se posent ainsi des cas d'école, des cas limites, et en inventent à loisir: la formule «je te baptise» est-elle valable dans le cas du baptême de frères siamois? Peut-elle être prononcée par un prêtre manchot, pendant qu'un prêtre muet procède à l'aspersion? Que penser, encore, de ce cas saugrenu : «Je te baptise au nom du Père - va chercher du vin - et du Fils, et du Saint-Esprit»? Plus réalistes sont les hypothèses de mariages forcés, entre gens privés de raison, ou de vœux prononcés à contrecœur; la résolution de ces problèmes vise à partager le rôle respectif de la lettre et de l'intention dans la prononciation des paroles sacramentelles. Un intérêt particulier est porté au sacrement eucharistique et à la formule de transsubstantiation hoc est corpus meum (ceci est mon corps), qui suscite des questionnements plus poussés et politiquement plus sensibles, puisqu'elle touche aux fondements mêmes de la religion chrétienne et aux principes de l'autorité de ses prêtres. En témoigne la violence inaugurale avec laquelle les thèses de Bérenger de Tours sont condamnées en 1059; celui-ci avait remis en cause la force opérative du sacrement en prouvant par des arguments grammaticaux et logiques - notamment par la distinction aristotélicienne de la substance et des accidents - que le Christ n'est présent qu'in figura, en figure, et pas en vérité dans l'hostie. La particularité de la conversion eucharistique, qui fait aussi sa fragilité dogmatique, est en effet d'être le seul acte pure-

1. Voir Pierre Bourdieu, Ce que parler veut dire. L'Économie des échanges linguistiques, Paris, Fayard, 1982. 
ment linguistique parmi les sacrements: son opérativité a pour seule et unique cause la formule consacrée. Aussi les réflexions sur ce sacrement exceptionnel doivent-elles nécessairement trouver dans des principes logiques et grammaticaux leur légitimité. Le problème de la deixis y est associé à celui des énoncés performatifs : il s'agit autant de savoir comment le pain est effectivement transformé en corps du Christ, que d'établir la cohérence entre la référence grammaticale du hoc, et sa référence réelle dans la situation d'énonciation.

L'histoire de la conception de l'efficacité sacramentelle est ainsi faite de controverses et de consensus, de compromis entre autorités en crise ou concurrentes. Les convictions religieuses tiennent leur puissance dogmatique non pas tant de l'autorité absolue du Texte originaire, que de leur lente élaboration dans une histoire accidentée, de l'articulation et de la confrontation d'autorités philosophiques. Ce qui rend passionnante la question médiévale des sacrements, c'est qu'elle montre à quel point la religion s'incarne dans l'histoire du concept: en livrant ses énoncés à la philosophie, elle catalyse l'articulation de domaines qui ne se seraient peut-être pas rencontrés sans elle: la logique, la grammaire et la théologie. Elle solidarise les nœuds les plus féconds de la réflexion sur le langage, et donc sur la pensée elle-même. Pour penser la performativité sacramentelle, les théologiens se rapportent librement aux arts libéraux, en entrelacent audacieusement les principes, comme autant d'outils mis à la disposition d'une cause qui les dépasse.

Pourtant, remarque Irène Rosier-Catach à l'issue de son impressionnant tour d'horizon, il n'existe pas d'examen des formules sacramentelles qui débouche sur la description des actes du langage en général, pas de théorie qui s'applique à d'autres types d'énoncés. La théologie, une fois affermies ses positions, se sépare à nouveau de la logique comme si l'idée d'une solidarité entre le discours religieux et le discours usuel, sur laquelle elle se fonde pour comprendre sa propre parole, n'avait plus de valeur. 